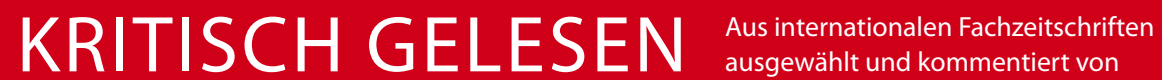

\begin{tabular}{|c|c|c|c|c|c|c|}
\hline $\begin{array}{l}\text { Prof. Dr. med. } \\
\text { H. S. FüeßI }\end{array}$ & $\begin{array}{l}\text { Prof. Dr. med. } \\
\text { H. Holzgreve }\end{array}$ & $\begin{array}{l}\text { Prof. Dr. med. } \\
\text { E. Ernst }\end{array}$ & $\begin{array}{l}\text { Prof. Dr. med. } \\
\text { K. Malberg }\end{array}$ & $\begin{array}{l}\text { Prof. Dr. med. } \\
\text { H.-C. Diener }\end{array}$ & $\begin{array}{l}\text { Prof. Dr. med. } \\
\text { P. E. H. Schwarz }\end{array}$ & $\begin{array}{l}\text { PD Dr. med. } \\
\text { T. M. Kapellen }\end{array}$ \\
\hline $\begin{array}{l}\text { Isar-Amper- } \\
\text { Klinikum, } \\
\text { Kl. München-Ost, } \\
\text { Haar }\end{array}$ & $\begin{array}{l}\text { Internist, } \\
\text { Kardiologische } \\
\text { Praxis, München }\end{array}$ & $\begin{array}{l}\text { Peninsular } \\
\text { Medical School, } \\
\text { University } \\
\text { of Exeter/UK }\end{array}$ & $\begin{array}{l}\text { Immunologie, } \\
\text { Dresden- } \\
\text { Loschwitz }\end{array}$ & $\begin{array}{l}\text { Klinik für } \\
\text { Neurologie, } \\
\text { Universitätsklinik } \\
\text { Essen }\end{array}$ & $\begin{array}{l}\text { Med. Klinik III, } \\
\text { TU Dresden }\end{array}$ & $\begin{array}{l}\text { Klinik und Poli- } \\
\text { klinik für Kinder } \\
\text { und Jugendliche, } \\
\text { Universität Leipzig }\end{array}$ \\
\hline
\end{tabular}

\section{Implantierte Defibrillatoren - Qual am Lebensende?}

Ein implantierter Defibrillator (ICD), der Patienten das Leben retten soll und dies mit adäquaten, elektrischen, schmerzhaften Schocks auch erreicht hat, kann am Lebensende zur Qual werden.

- Der Autor machte 14 Studien ausfindig, die sich mit der Deaktivierung von ICD's am Lebensende beschäftigen. Nur 27\% der Angehörigen von Verstorbenen gaben an, dass vor dem Tod über eine Deaktivierung gesprochen wurde. Selbst bei Patienten mit einer DNR-Verfügung (keine Wiederbelebung) wurde

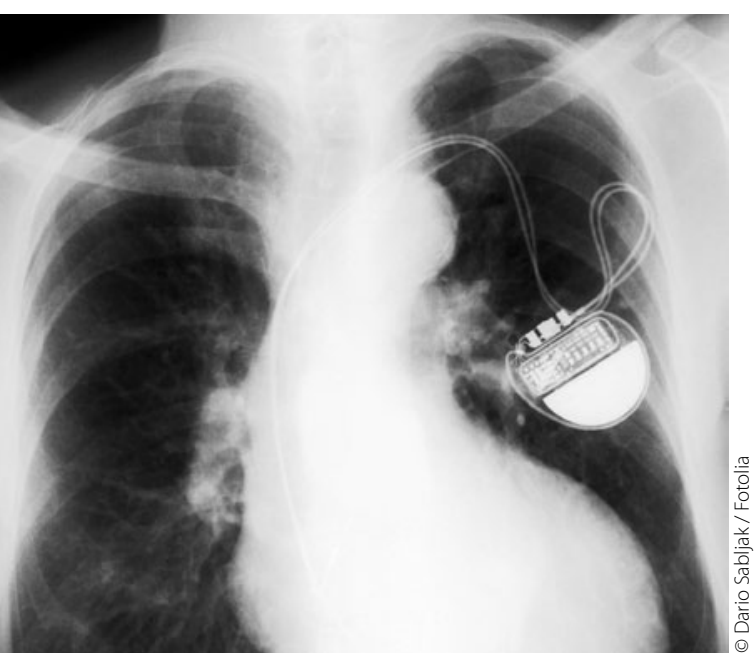

ICD: 33 Schocks bei einem Sterbenden. dieses Thema nicht einmal mit jedem 2. Patienten und seinen Angehörigen angesprochen. Nur 25\% der Internisten und Hausärzte bzw. 40\% der Geriater thematisierten diese Option.

Das Thema ist äußerst komplex. Die Mehrzahl der Ärzte befürwortet eine Information der Patienten über die Möglichkeit einer Deaktivierung und deren Gründe schon zum Zeitpunkt der Implantation. Sie unterstellen auch, dass die Träger von ICD's und ihre Angehörigen ausreichend informiert sind. Doch die Patienten und ihre Angehörigen beklagen, dass sie die Technik und ihre
Probleme unzureichend verstehen und erwarten, dass die behandelnden Ärzte geeignete Maßnahmen vorschlagen. Die Ärzte wiederum scheuen sich, dieses delikate Thema bei ihren Patienten anzusprechen, zumal viele Patienten die Bedeutung dieser Geräte am Lebensende überschätzen, häufig genug eine komplexe psychologische Beziehung zu dieser Technik entwickelt haben und deren Funktion erhalten möchten.

\section{- J. E. Russo}

Deactivation of ICD's at the end of life. Amer. J. Nursing 111 (2011) 26-35

\section{Kommentar}

$I C D$ 's sind die Standardtherapie bei lebensbedrohlichen Herzrhythmusstörungen. Weil sie den Herztod infolge Rhythmusstörungen erfolgreich verhindern, nähern sich viele solcher Patienten ihrem Sterbeprozess infolge ganz anderer, nur palliativ behandelbarer Krankheiten. Die Diskussion um die Deaktivierung von ICD's am Lebensende wurde durch einen schockierenden Leserbrief an eine Fachzeitschrift angestoßen: Ein Patient wurde mit funktionstüchtigem ICD in häusliche palliative Pflege verlegt. Die Ehefrau berichtete, dass ihr Mann in ihren Armen verstorben sei, während das Gerät 33 Schocks abgab und sich so erhitzte, dass die
Haut verbrannte. Seither wird dieses Thema intensiv diskutiert. Es gibt inzwischen auch Leitlinien und detaillierte Protokolle von Fachgesellschaften für die Deaktivierung von ICD's. Trotzdem bleibt die Abschaltung ein heikles Problem für jeden Arzt, der einen Sterbenden betreut. Er muss seinem Patienten erklären, dass das Gerät, das ihm als Lebensretter empfohlen wurde, nun plötzlich abgestellt werden soll. Und dann hat er nicht die Erfahrungen und die Mittel (Magnet) für die Deaktivierung und muss einen Mitarbeiter der Herstellerfirma oder einen Kollegen aus der Kardiologie zum Hausbesuch bitten.

H. HolzGREVE . 